Monatsschr Kinderheilkd 2021 • 169:467-469 https://doi.org/10.1007/s00112-021-01170-4 Angenommen: 9. März 2021

Online publiziert: 8. April 2021

(c) Springer Medizin Verlag GmbH, ein Teil von Springer Nature 2021

\section{Redaktion}

R. Berner, Dresden

G. Hansen, Hannover

R. Kerbl, Leoben

F. Zepp, Mainz

\section{Leserbrief zu}

Jung P, Mileder L, Hoffmann F et al (2020) Simulationsbasierte pädiatrische Notfallteamtrainings in Zeiten der SARS-CoV-2-Pandemie. Monatsschr Kinderheilkd 168:1130-1131. https://doi.org/10.1007/s00112-020-01055-y

\section{Replik}

Mileder L, Jung $P$, Hoffmann F, Heimberg $E$ (2021) Gemeinsam sicher für unsere kleinen und kleinsten Patient ${ }^{*}$ innen trainieren. Monatsschr Kinderheilkd. https://doi.org/10. 1007/s00112-021-01171-3

Sehr geehrte Autorinnen und Autoren, liebe Freundinnen und Freunde,

die durch die "coronavirus disease 2019“ (COVID-19) verursachte Pandemie hat die Welt weiterhin fest im Griff, und es wird derzeit besonders deutlich, welchen hohen Stellenwert gut ausgebildetes und trainiertes medizinisches Personal für die Bewältigung dieser schwersten medizinischen, wirtschaftlichen und gesellschaftlichen Krise seit dem 2. Weltkrieg hat.

Mit großem Interesse haben wir daher die Stellungnahme „Simulationsbasierte pädiatrische Notfalltrainings in Zeiten der SARS-CoV-2-Pandemie" in der Novemberausgabe der Monatsschrift Kinderheilkunde gelesen. Wir können den meisten der Punkte, die die von uns sehr geschätzten Kolleg*innen dargelegt haben, vorbehaltlos zustimmen. So stimmen wir selbstverständlich uneingeschränkt zu, dass die „bestmögliche

\author{
Jens-Christian Schwindt ${ }^{1}$ Hans Härting ${ }^{2}$ \\ ' SIMCharacters Training GmbH, Wien, Österreich \\ ${ }^{2}$ AssekuRisk Safety Management $\mathrm{GmbH}$, Wien, Österreich
}

\title{
Dringender Appell zu gesetzlich geregelten Sicherheits- und Trainingsstandards
}

Bewältigung eines medizinischen Notfalls von vielen Faktoren abhängig ist, v. a. aber auch von der guten und effektiven Zusammenarbeit des versorgenden Behandlungsteams“ [1]. Gewundert haben wir uns jedoch über die Schlussfolgerung in der Stellungnahme, dass es den Kolleg*innen „als Ärzten und Simulationstrainern schwerfällt, eine klare und ausschließliche Empfehlung für die Wiederaufnahme des regulären Trainingsbetriebs zu geben oder diese gar zu fordern."

Hierzu möchten wir, ebenfalls als Ärzte, Patientensicherheitsexperten, Simulationstrainer und Anbieter von Simulations- und Patientensicherheitstrainings, unsere Gedanken kurz darlegen.

In zahlreichen Hochsicherheitsorganisationen, wie z.B. der kommerziellen Luftfahrt, sind Teamsimulationstrainings fester und gesetzlich vorgeschriebener Bestandteil der Aus- und Weiterbildung und damit eine vom Gesetzgeber festgelegte entscheidende Säule der betrieblichen Sicherheit. Auch die Trainingsintervalle der Crews sind gesetzlich vorgeschrieben und stellen eine Grundlage der Erlaubnis zur Berufsausübung bzw. für die Betriebserlaubnis der Fluggesellschaft dar. Während der Pandemie haben zahlreiche Fluggesellschaften ihren Flugbetrieb minimieren müssen und teilweise sogar komplett eingestellt. Die gesetzlich vorgeschriebenen Fristen für notwendige Trainings der Crews wurden daher geringfügig verlängert. Die Airlines sind jedoch verpflichtet, die Trainings und Checks der Crews innerhalb dieser festgelegten Intervalle zu gewähr leisten, da die Berechtigung der Crews zum Betrieb eines Flugzeuges in Cockpit oder Kabine ohne die vorgeschriebenen Trainings, auch in Pandemiezeiten, erlöschen würde. Mit der Wiederaufnahme und Intensivierung des Flugbetriebes müssen darüber hinaus gesetzlich erforderliche "requalification trainings" in einem festgelegten Ausmaß von jedem einzelnen Crew-Mitglied vollständig absolviert werden, um die Berechtigung zur Berufsausübung aufrechtzuerhalten bzw. wiederzuerlangen.

Im Gegensatz zur Luftfahrt wurde die medizinische Versorgung während der COVID-19-Pandemie nicht eingestellt, sondern musste, im Gegenteil, pandemiebedingt in vielen Bereichen sogar intensiviert werden, verbunden mit einer erheblichen zusätzlichen Arbeitsbelastung (und emotionalen Belastung!) für das betroffene medizinische Personal. Zunächst war teilweise zu beobachten, dass in Kliniken, in denen Training bereits einen Stellenwert vor der Pandemie hatte, zusätzliche Trainings zur speziellen Versorgung von COVID-19-Patienten durchgeführt wurden. Im weiteren Verlauf wurden jedoch, v. a. aus Sorge vor Infektionsübertragungen während des Trainings, zahlreiche Reanimations- und Simulationstrainings in Kliniken vorsorglich abgesagt. In der Luftfahrt wäre es, wie oben beschrieben undenkbar, dass aus Angst vor einer Ansteckung der Crew-Mitglieder im Rahmen eines Notfalltrainings Trainingseinheiten während einer Pandemie nicht durchge- 
führt würden, da die Team-Performance im Notfall nachgewiesenermaßen u.a. von regelmäßigem Simulationstraining im Team abhängig ist [2].

Haben also medizinische Simulationstrainings demgegenüber einen so geringen Stellenwert für die Performance von medizinischen Teams, dass wir es uns tatsächlich leisten können, medizinische Teamtrainings während einer Pandemie auszusetzen?

Dass es selbst Expert*innen im Bereich Simulationstraining und Patientensicherheit allerdings offensichtlich schwerfällt, sich auch in einer Pandemie klar und eindeutig für die Fortführung von simulationsbasierten Teamtrainings auszusprechen, hat nach unserer Ansicht mehrere Gründe:

- Teamsimulationstraining wird in der Medizin weiterhin eben nicht als unverzichtbarer Bestandteil der Ausund Weiterbildung angesehen. Die Durchführung und das Angebot von Teamsimulationstrainings hängen weiterhin vorwiegend vom Engagement motivierter Einzelpersonen $\mathrm{ab}$.

- Training hat weiterhin nicht den gleichen Stellenwert wie das klinische Arbeiten; eine Ansteckung im Rahmen eines Trainings würde daher wahrscheinlich derzeit anders bewertet, als wenn diese während des klinischen Arbeitens erfolgt, und deswegen eher als „unnötig“ angesehen.

- Die Durchführung von simulationsbasierten Teamtrainings in der Medizin zur Gewährleistung der Patient ${ }^{*}$ innen- und Mitarbeiter ${ }^{*}$ innensicherheit ist weiterhin nicht gesetzlich geregelt. Die Entscheidung, ob Trainings stattfinden oder nicht, hängt damit nicht von gesetzlich festgelegten Erfordernissen ab, was Stellungnahmen, wie die vom Netzwerk Kindersimulation und PAEDSIM e. V., überhaupt erst notwendig macht.

Dass es in einem so sicherheitssensiblen Bereich wie der Medizin noch keine gesetzlichen Mindesterfordernisse für Notfalltrainings gibt, ist nach aktuellem Wissensstand und im Vergleich $\mathrm{zu}$ ande- ren Hochsicherheitssystemen nicht akzeptabel. Das durch fehlende Trainings entstehende Risiko wird auf die Mitarbeiter*innen und Patient ${ }^{*}$ innen übertragen. Risikoeigner ist die Organisation, die zum Schutz der Patient*innen und Mitarbeiter*innen Trainings nach eigenem Ermessen und Budget durchführen kann oder nicht. Dies ist z. B. im System Luftfahrt unvorstellbar, denn hier verhindern klar festgelegte gesetzliche Trainingsintervalle, dass Sicherheitsstandards aufgrund von Konkurrenzdruck oder einer Fehleinschätzung der Risiken von Fluggesellschaften unterlaufen werden könnten.

In beiden Systemen bedeutet ein Trainingsdefizit aber gleichermaßen ein Risiko für die betroffenen Personen (Patient*innen, Pflegekräfte und Ärzt*innen, Crews und Passagiere, [2]). Ist es tatsächlich vorstellbar, dass Passagiere einen Verzicht auf das Training der Pilot ${ }^{\star}$ innen und Flugbegleiter*innen wegen der damit verbundenen möglichen Infektionsgefahr akzeptieren würden, und das so entstehende Sicherheitsrisiko letztlich einfach in Kauf genommen werden muss?

Wenn wir also auch medizinisches Simulationstraining als unverzichtbaren Bestandteil der Aus- und Weiterbildung von medizinischen Teams und als eine entscheidende Säule der innerbetrieblichen Sicherheit in Kliniken ansehen und insbesondere dem Training von seltenen Notfallsituationen den gleichen Stellenwert wie der klinischen Arbeit beimessen, bleibt aus unserer Sicht keine andere Möglichkeit, als sich auch während einer Pandemie klar für die Fortführung von medizinischen Simulationstrainings auszusprechen. Selbstverständlich müssen die Trainings, angepasst an die Pandemieaktivität, unter besonderen hygienischen Sicherheitsmaßnahmen (z.B. Masken der „Filtering-face-piece“[FFP]Kategorie 2 für Trainer*innen und Teilnehmer*innen) durchgeführt werden. Theoretische Trainingsinhalte sollten, in Abhängigkeit von der Pandemielage, großzügig in den Onlinebereich verlagert werden, um die direkte Kontaktzeit der Trainingsteilnehmer*innen während der unverzichtbaren praktischen Handson-Trainingsteile auf das tatsächlich notwendige zu beschränken [3]. Solange jedoch die akute Patientenversorgung aufgrund z.B. eines zu befürchtenden kritischen Ausfalls von medizinischem Personal oder einer massiven Überlastung nicht gefährdet ist, muss aus unserer Sicht auch die Durchführung von Teamsimulationstrainings während einer Pandemie möglich sein. Auch wir hoffen dabei natürlich auf die Impfstoffe und eine möglichst rasche und lückenlose Durchimpfung des medizinischen Personals, um Trainings auch auf dieser Basis wieder sicher durchführen $\mathrm{zu}$ können.

Zusammenfassend hätten wir uns daher gerade vom Netzwerk Kindersimulation e.V. und PAEDSIM e.V., deren Vereinszwecke in der Erhöhung der $\mathrm{Pa}$ tientensicherheit durch medizinisches Simulationstraining für pädiatrische $\mathrm{Pa}$ tient*innen liegen, eine klare Empfehlung für die Durchführung von simulationsbasierten Teamtrainings auch während einer Pandemie gewünscht.

Wir sind uns durchaus bewusst, dass wir uns, wenn wir uns als Anbieter von Simulations- und Patientensicherheitstrainings für die Fortführung von Teamsimulationstrainings auch während einer Pandemie einsetzen, natürlich einen Interessenkonflikt vorwerfen lassen müssen. Wir sind eben auch Ärzte, Simulationstrainer und Experten für Patient ${ }^{*}$ innen- und Mitarbeiter*innensicherheit und fühlen uns daher verpflichtet, unsere Einschätzung zu diesem Thema abzugeben. Nach unserer Auffassung ist es nun mehr denn je notwendig, klare gesetzlich geregelte Sicherheits- und Trainingsstandards, analog zu anderen Hochzuverlässigkeitsorganisationen, auch für das medizinische System, zu fordern. Nur durch eine gesetzliche Regelung lässt sich jedem gegenläufigen Konkurrenzdruck und jeder Fehleinschätzung von Risiken, auch im medizinischen System, grundsätzlich vorzubeugen.

Die aktuelle Situation wäre eine gute Gelegenheit für das Netzwerk Kindersimulation e. V. und PAEDSIM e. V. gewesen, um die Diskussion anzustoßen, welchen Stellenwert Training von medizinischem Personal in der Versorgung von kritisch kranken Kindern, nicht nur 
während einer Pandemie hat, sondern bisher grundsätzlich hatte und v.a. in Zukunft haben soll. Auch nach dem Ende der Pandemie muss diese Diskussion erneut aufgenommen und zielorientiert fortgeführt werden - zum Wohle der uns anvertrauten Kinder, ihrer Familien und der Mitarbeiterinnen und Mitarbeiter im medizinischen System.

\section{Korrespondenzadresse}

\section{Dr. Jens-Christian Schwindt}

SIMCharacters Training GmbH

Wien, Österreich

jens.schwindt@simcharacters.com

Dr. Jens-Christian Schwindt ist Geschäftsführer der SIMCharacters Training GmbH, Wien, Neonatologe, PAEDSIM-Gründungsmitglied und Mitglied des Vorstandes des Austrian Resuscitation Council (ARC).

Capt. Hans Härting ist Geschäftsführer der AssekuRisk Safety Management GmbH, Wien, Kapitän auf einem Airbus A320 einer großen österreichischen Airline, verantwortlich für die "crew safety training standards" eines großen europäischen Airline-Verbundes.

Interessenkonflikt. J.-C. Schwindt ist Geschäftsführer von SIMCharacters Training GmbH (Wien, Österreich), eines Anbieters von pädiatrischen Reanimations- und Simulationstrainings. H. Härting ist Geschäftsführer der AssekuRisk Safety Management GmbH (Wien, Österreich), eines Anbieters von Sicherheitstrainings.

\section{Literatur}

1. Schmutz J, Manser T (2013) Do team processes really have an effect on clinical performance? A systematic literature review. $\mathrm{Br} J$ Anaesth 110(4):529-544

2. Müller M (2003) Risikomanagement und Sicherheitsstrategien der Luftfahrt - ein Vorbild für die Medizin? ZAllg Med 79:339-344

3. Schwindt J, Pflanzl-Knizacek L, Nierscher FJ, Hoffmann P, Singraber E, Cardona F, Burda G, Koestenberger M, Egger A, Schlieber J, Baubin $M$ Durchführung von Kursen zur Aus- und Fortbildung während der COVID-19-Pandemie. www.arc.or.at/covid/.Zugegriffen:11.Dez. 2021

\section{Neue Sint1a-Studie will Entstehung von Typ-1-Diabetes bei Kindern verhindern}

Die Wahrscheinlichkeit bis zum 18. Lebensjahr an Typ-1-Diabetes zu erkranken liegt bei etwa 1 zu 300. Rund $90 \%$ der betroffenen Kinder und Jugendliche haben keinen nahen Verwandten mit der Autoimmunkrankheit, weshalb die Diagnose oft überraschend kommt. Bei Personen mit Typ-1Diabetes zerstört das Immunsystem die insulinproduzierenden Zellen in den "Inseln" der Bauchspeicheldrüse. Betroffene müssen sich ein Leben lang Insulin spritzen. Insulin hat eine lebenswichtige Funktion: Es transportiert den Zucker aus dem Blut in die Körperzellen. Meist ist das körpereigene Insulin das erste Ziel der Immunreaktion, die zu Typ-1Diabetes führt.

In frühen Stadien von Typ-1-Diabetes treten sogenannte Inselautoantikörper im Blut auf. Sie sind ein Anzeichen dafür, dass das Immunsystem die insulinproduzierenden Zellen angreift. Aus früheren Studien wissen die Forschenden, dass die Darmflora bei Kindern, die diese Antikörper entwickeln, gestört sein kann. Die neue Sint1a-Studie zielt darauf ab, das Auftreten von Inselautoantikörpern bei Kindern mit einem erhöhten genetischen Risiko für Typ-1-Diabetes zu verhindern. Dazu wird innen das Probiotikum Bifidobacterium Infantis (aktiviertes B. infantis EVC001) zusammen mit der täglichen Nahrung verabreicht. Dies soll eine gesunde und ausgewogene Entwicklung ihrer Darmflora unterstützen. Dadurch könnte das Immunsystem, noch bevor erste Anzeichen von Autoimmunität auftreten, positiv beeinflusst werden.

Sint1a baut auf eine andere Studie namens Point auf. In der Point-Studie erhalten Kinder Insulinpulver, das ebenfalls gemeinsam mit der Nahrung verabreicht wird. Ziel ist es, das Immunsystem frühzeitig zu trainieren und zu für Insulin sensibilisieren, damit keine Autoimmunität gegen das Hormon entsteht. „Wir gehen davon aus, dass das Immunsystem der Mund- und Darmschleimhaut für die Prävention von Krankheiten wie Typ-1Diabetes eine wichtige Rolle spielt. Die PointStudie nutzt den Darm (wo das Insulinpulver ankommt), um das Immunsystem mit Insulin vertraut zu machen und eine Autoimmunreaktion dagegen zu verhindern. Sint 1 a basiert auf dem Wissen, dass eine gesunde Darm- flora Entzündungen reduziert und dies dem Immunsystem hilft, gefährliche Antigene von ungefährlichen zu unterscheiden", erklärt Studienleiterin Anette Ziegler. Auf diese Weise will die Sint1a-Studie die Wahrscheinlichkeit verringern, dass Kinder mit einem hohen genetischen Risiko für Typ-1-Diabetes, Immunreaktionen auslösen, die zu einer Autoimmunität führen. "Sollten die Ergebnisse beider Studien erfolgreich sein", erklärt Ziegler, „werden wir sie für eine optimierte synergistische Typ-1-Diabetes-Präventionsstrategie miteinander kombinieren. Typ-1Diabetes wäre dann kein unausweichliches Schicksal mehr, sondern eine Krankheit, die wir mit den richtigen Maßnahmen verhindern können.

Die Sint1a-Studie läuft ab April 2021 in mehreren europäischen Ländern im Rahmen von GPPAD (Global Platform for the Prevention of Autoimmune Diabetes), einer internationalen Initiative zur Prävention von Typ1-Diabetes. Die GPPAD-Forschungszentren in Belgien (Leuven), Deutschland (Dresden, Hannover, München), Großbritannien (Cambridge, Newcastle), Polen (Warschau) und Schweden (Malmö) liefern die dafür notwendige wissenschaftliche Infrastruktur. Internationale populationsbasierte Typ-1Diabetes-Präventionsstudien wie diese sind weltweit einzigartig.

Die Teilnahme an den Sint1a- und PointStudien erfordert den Nachweis eines erhöhten genetischen Typ-1-Diabetes-Risikos. Ein Früherkennungstest ermöglicht es, dieses Risiko lange vor der Manifestation der Krankheit zu bestimmen. Der Test kann mit wenigen Blutstropfen innerhalb der ersten 7 Lebenstage durchgeführt werden. Anette Ziegler und ihr Team haben dazu im Jahr 2016 eine Screening-Studie ins Leben gerufen, die in Deutschland unter dem Namen „Freder1k“ läuft. „Innerhalb des länderübergreifenden GPPAD-Netzwerks konnten wir bereits 245.000 Babys untersuchen. Bei 1,15 $\%$ haben wir ein erhöhtes genetisches Risiko festgestellt. Die Früherkennung ist das $A$ und 0 , um sinnvolle Präventionsmaßnahmen zu ergreifen", sagt Ziegler.

Quelle: www.helmholtz-muenchen.de 